\title{
Better adherence to prescribed treatment regimen is related to less chronic pain among adolescents and young adults with moderate or severe haemophilia
}

\author{
J. M. MCLAUGHLIN, $*$ M. L. WITKOP,† A. LAMBING, $\neq$ T. L. ANDERSON, $*$ J. MUNN $\$ and \\ B. TORTELLA* \\ *Medicines Development Group, Pfizer Specialty Care, Collegeville, PA; †Northern Regional Bleeding Disorders Center, \\ Munson Medical Center, Traverse City; $\$$ Adult Hemophilia \& Thrombosis Treatment Center, Henry Ford Health System, \\ Detroit; and \$Hemophilia Treatment Center, University of Michigan, Ann Arbor, MI, USA
}

Summary. Little data exist, especially for adolescent and young adult (AYA) persons with haemophilia (PWH), about the relationship between adherence to prescribed treatment regimen and chronic pain. We examined this relationship among PWH (moderate or severe) aged 13-25 via cross-sectional survey. Adherence was assessed using the Validated Hemophilia Regimen Treatment Adherence Scale (VERITAS)-Pro and VERITAS-PRN for prophylactic and on-demand participants respectively. VERITAS scores range from 24 (most adherent) to 120 (least adherent). Chronic pain was measured using the FPS-R and was dichotomized as high for FPS-R scores $\geq 4$ and low for $<4$. Logistic regression models were constructed to assess factors associated with having high (vs. low) chronic pain. Of 80 AYA respondents (79 men), most had severe disease $(91 \%)$, infused prophylactically $(86 \%)$ and had haemophilia A $(91 \%)$. Fifty-one per cent were aged 13-17 and most were white $(76 \%)$, non-Hispanic $(88 \%)$ and never married $(93 \%)$. Chronic pain was reported as high for $35 \%$ of respondents. Mean VERITAS-Pro scores for those with high and low chronic pain were $53.6 \pm 12.3$ vs. $47.4 \pm 12.9, P=0.05$. VERITAS-PRN scores were similar across chronic pain status. Logistic regression revealed that for each 10-point reduction (i.e. increase in adherence) in the combined VERITAS (Pro and PRN) and VERITAS-Pro scores there was a $35 \%$ $(\mathrm{OR}=0.65 ; 95 \% \mathrm{CI}=0.44,0.96 ; P=0.03)$ and $39 \%$ $(\mathrm{OR}=0.61 ; 95 \% \mathrm{CI}=0.39,0.96 ; P=0.03)$ reduction in odds of having high chronic pain respectively. Among AYA PWHs, better adherence was associated with significantly lower odds of having high chronic pain. Moreover, non-whites were $>4$ times as likely as whites to report high chronic pain.

Keywords: adherence, adolescents, chronic pain, clotting factor, haemophilia, young adults

\section{Introduction}

Little data exist, especially for adolescents and young adults (AYA), about the relationship between level of adherence to prescribed haemophilia treatment regimens and chronic pain. Practical experience with most haemophilia patients has demonstrated that adherence to both on-demand and prophylactic treatment regimens, although variable, is less than ideal. According to the National Hemophilia Foundation

Correspondence: John M. McLaughlin, PhD, MSPH, Director, Institutional Outcomes Research Scientist, US Medical Affairs, Pfizer Inc, New York, NY, USA.

Tel.: (614) 505 6142; fax: (646) 348 8228;

e-mail: john.mclaughlin@pfizer.com

Accepted after revision 9 December 2013
(NHF), nearly half of all patients and parents of patients reported that bleeds were not treated early on [1]. Other reports have shown that approximately $40 \%$ of patients reported not following prescribed treatment regimens [2] or rated their adherence as less than excellent [3]. Consequently, many patients experience serious complications stemming from poor adherence [3-7]. Specifically, poor adherence to prescribed clotting-factor treatment regimens can lead to worsened and repeated bleeding episodes in the joints, muscles and/or soft tissue [5]. Over time these episodes can lead to complications including haemarthropathy, which often manifests itself as debilitating chronic pain. To help quantify the relationship between adherence and health outcomes among persons with haemophilia (PWH), several studies have attempted to measure and/or evaluate the impact of 
adherence [2,3,7-9]. However, because a standardized and validated measure of adherence was not used in previous studies, contextualizing and comparing results have not been possible. Admittedly, previous authors have recognized this fact $[2,3,8,9]$. Moreover, data describing adherence among AYA PWH are particularly lacking. AYAs are a unique population of $\mathrm{PWH}$ who are often (i) just starting to take more responsibility for the management of their own disease and (ii) developing treatment habits that can carry over into adult life [2]. To that end, the primary aim of this study was to evaluate the relationship between a newly validated measure of adherence to prescribed clotting-factor treatment regimens and chronic pain among AYAs with moderate or severe haemophilia using data collected via a large cross-sectional survey administered in 2012.

\section{Materials and methods}

\section{Study population and recruitment}

Data describing PWH's adherence to prescribed treatment regimens and level of chronic pain were obtained as part of the larger Interrelationship between Management of Pain, Adherence to Clottingfactor Treatment and Quality of Life (IMPACT QoL) study. As the name suggests, the IMPACT QoL study had the primary goal of assessing the relationship between validated measures of pain, clotting-factor adherence and QoL among AYA PWHs and AYAs with von Willebrand disease (VWD). In addition, as part of the IMPACT QoL study we also evaluated how similar an AYA patient's perceptions of pain, adherence and QoL were compared to the perceptions of their carer (e.g. parent or spouse) and their health care provider. Data were collected via a one-time, cross-sectional, online survey from a convenience sample of AYA patients with a bleeding disorder. To be eligible to complete the survey, participants had to (i) be aged 13-25 years, (ii) read, write and speak English, and (iii) have haemophilia A, haemophilia B, or VWD. Recruitment occurred at major US haemophilia meetings (e.g. Inhibitor Summits and NHF meetings), US haemophilia treatment centres (HTC), and through a Facebook $^{\text {TM }}$ (Facebook, Menlo Park, CA, USA) page dedicated to the study from April to December of 2012. All surveys were completed electronically using SurveyMonkey ${ }^{\mathrm{TM}}$ (SurveyMonkey, Palo Alto, CA, USA) and Apple iPads ${ }^{\mathrm{TM}}$ (Apple, Cupertino, CA, USA). The study was approved by the Munson Medical Center (Traverse City, MI, USA) institutional review board prior to data collection. All data were de-identified prior to analysis. This study uses a patient subset of the IMPACT QoL survey data to analyse the relationship between adherence to clotting-factor treatment and chronic pain among AYAs with moderate or severe haemophilia. Patients with mild haemophilia or VWD were excluded from this analysis because of small sample size and to minimize heterogeneity in the assessment of the primary hypothesis.

\section{Measurement}

Adherence was assessed using the Validated Hemophilia Regimen Treatment Adherence Scale (VERITAS)-Pro [10] and VERITAS-PRN [11] for prophylactic and on-demand (i.e. episodic) participants respectively. VERITAS scores range from 24 (most adherent) to 120 (least adherent). As an experimental measure, we also combined VERITAS-Pro and VERITAS-PRN responses into one category (VERITAS-combined) to evaluate the relationship between adherence and chronic pain for both prophylactic and on-demand AYA PWH simultaneously. Chronic pain was measured using the revised Faces Pain Scale (FPS$\mathrm{R})$. The FPS-R is a visual scale composed of six faces illustrating an increasing level of pain intensity. Respondents were asked to choose the face that best describes the intensity of the chronic pain they experienced. In the IMPACT QoL survey, chronic pain was defined as 'pain that you have every day or almost every day, and that always or almost always seems to be there even when you are not having a bleed at that moment.' FPS-R scores range from 0 to 10 with the faces representing the lowest and highest levels of pain intensity coded as 0 and 10 respectively. The FPS-R is highly correlated with the visual analog scale $(r=0.93)$ and with the coloured analog scale $(r=0.84)$, demonstrating strong validity. Moreover, reliability and validity of the FPS-R have been established for a broad age range, ranging from children as young as 4 years old to adults [12]. For the purpose of analysis, chronic pain was dichotomized as high for those who reported their pain as 'moderate', 'severe', 'very severe', or 'worst pain possible' (i.e. $\geq 4$ ) and low for 'mild pain' or 'no pain' (i.e. <4).

\section{Statistical analysis}

Descriptive statistics and univariate relationships were assessed by tabulating chronic pain status by VERITAS scores and patient sociodemographic and clinical characteristics. Percentages were used to describe categorical variables and statistical association was assessed using Fisher's exact test because of small sample size. Multivariable, parsimonious logistic regression models were constructed to assess factors associated with having high (vs. low) levels of chronic pain. Separate models were constructed to evaluate (i) a combined, experimental VERITAS score among prophylactic and on-demand patients and (ii) the VERITAS-Pro score among prophylactic patients only. 
Small sample size prevented analysis for on-demand (only) participants. Due to the large number of variables collected as part of the survey and because of the small sample size inherent in rare disease research, in addition to the fully adjusted models, final parsimonious models were constructed. In the final parsimonious models, we decided, a priori, to model VERITAS score as the primary variable of interest and include other covariates in the model only if they (i) changed the odds ratio (OR) of the VERITAS score parameter by at least $10-15 \%$ (i.e. confounded) [13], (ii) improved the precision of the estimated VERITAS score parameter, or (iii) were statistically significant at a two-tailed alpha level of 0.05. All statistical analyses were performed using SAS 9.2 (SAS, Cary, NC, USA). All $P$-values were calculated using two-sided tests.

\section{Results}

Ninety-three AYAs with haemophilia completed the survey. Mild patients $(n=13)$ were excluded. Of the 80 participants $(79$ men) included in the final analysis, most had severe disease $(91 \%)$, infused prophylactically $(86 \%)$ and had haemophilia A (91\%). Fifty-one per cent were aged 13-17 years and most were white $(76 \%)$, non-Hispanic $(88 \%)$ and never married $(93 \%)$. Most $(73 \%)$ of non-white respondents were black or African American, 14\% were mixed race, $9 \%$ were Asian and $5 \%$ were American Indian or Alaskan Native. The majority (94\%) had some type of health insurance, with the highest proportion having only commercial insurance $(45 \%)$, only Medicaid $(28 \%)$, or both $(9 \%)$.

Mean VERITAS-Pro $(n=69)$ and PRN $(n=11)$ scores were $49.6 \pm 12.9$ (range 25-78) and $51.0 \pm 11.6$ (range 35-74) respectively. Chronic pain was reported as high for $35 \%$ of respondents. The proportion of respondents who reported having high levels of chronic pain was similar across treatment regimen $(36 \%$ for prophylactic vs. $27 \%$ for on demand, $P=0.74)$. At the univariate level, patients with self-reported high levels of chronic pain were more likely than those who reported low levels of pain to be young adults (vs. adolescents, $P=0.04$ ), be nonwhite $(P=0.01)$ and have government-provided health insurance or be uninsured (vs. commercial health insurance, $P=0.03$ ) (Table 1). Compared to those with low self-reported chronic pain, those with high chronic pain had higher (worse adherence) VERITAS-Combined scores $(53.1 \pm 12.0$ vs. $48.0 \pm 12.8$, $P=0.08)$, though not statistically significant at $P<0.05$. Among prophylactic respondents only, mean VERITAS-Pro scores for those with high chronic pain were higher (worse adherence) compared to those who reported low chronic pain $(53.6 \pm 12.3$ vs. $47.4 \pm 12.9, P=0.05)$. VERITAS-PRN scores were
Table 1. Self-reported respondent characteristics by chronic pain status $(n=80)$.

\begin{tabular}{|c|c|c|c|}
\hline Characteristic & $\begin{array}{c}\text { Low chronic } \\
\text { pain, } n(\%) \\
n=52\end{array}$ & $\begin{array}{c}\text { High chronic } \\
\text { pain, } n(\%) \\
n=28\end{array}$ & $\begin{array}{l}\text { Fisher's } \\
P \text {-value }\end{array}$ \\
\hline Age & & & 0.04 \\
\hline $13-17$ & $31(60)$ & $10(36)$ & \\
\hline $18-25$ & $21(40)$ & $18(64)$ & \\
\hline Gender & & & 0.35 \\
\hline Male & $52(100)$ & $27(96)$ & \\
\hline Female & $0(0)$ & $1(4)$ & \\
\hline Race & & & 0.01 \\
\hline White (only) & $45(87)$ & $16(57)$ & \\
\hline Non-white* & $7(13)$ & $12(43)$ & \\
\hline Ethnicity & & & 0.73 \\
\hline Hispanic & $6(14)$ & $5(12)$ & \\
\hline Non-Hispanic & $46(86)$ & $38(88)$ & \\
\hline Health insurance $^{\dagger}$ & & & 0.03 \\
\hline Medicaid or VA only ${ }^{\ddagger}$ & $12(24)$ & $12(43)$ & \\
\hline Commercial only & $27(54)$ & $8(29)$ & \\
\hline Both & $4(8)$ & $3(11)$ & \\
\hline $\begin{array}{l}\text { Insured type } \\
\text { unknown }\end{array}$ & $7(14)$ & $2(7)$ & \\
\hline Uninsured & $0(0)$ & $3(11)$ & \\
\hline Bleeding disorder & & & 0.31 \\
\hline Haemophilia A & $47(90)$ & $26(93)$ & \\
\hline Haemophilia B & $5(10)$ & $2(7)$ & \\
\hline Severity & & & 0.31 \\
\hline Moderate & $5(10)$ & $2(7)$ & \\
\hline Severe & $47(90)$ & $26(93)$ & \\
\hline Inhibitor development & & & 0.36 \\
\hline Ever & $22(42)$ & $15(54)$ & \\
\hline Never & $30(58)$ & $13(46)$ & \\
\hline Treatment regimen & & & 0.74 \\
\hline On demand & $8(15)$ & $3(11)$ & \\
\hline Prophylaxis & $44(85)$ & $25(89)$ & \\
\hline
\end{tabular}

"Most $(73 \%)$ of non-white respondents were black or African American, $14 \%$ were mixed race, $9 \%$ were Asian and $5 \%$ were American Indian or Alaskan native.

${ }^{\dagger} n=78$, two patients answered 'Don't Know' to whether or not they had health insurance and were not included.

¥Only two patients had VA only insurance, the others had Medicaid only.

similar across chronic pain status, though sample size was small $(n=11)$. Figure 1 compares VERITAS scores by chronic pain level.

Adjusted logistic regression analysis revealed that for each 10-point reduction (i.e. increase in adherence) in the combined, experimental VERITAS score (Pro and $\mathrm{PRN})$ there was a $35 \% \quad(\mathrm{OR}=0.65 ; 95 \%$ $\mathrm{CI}=0.44,0.96 ; P=0.03)$ reduction in the odds of having high chronic pain. Among prophylactic respondents only, for each 10-point reduction in the VERITAS-Pro score there was a $39 \%(\mathrm{OR}=0.61 ; 95 \%$ $\mathrm{CI}=0.39,0.96 ; P=0.03)$ reduction in the odds of having high chronic pain (Tables 2 and 3). Among all and prophylactic-only respondents, respectively, the model also revealed that, compared to whites, nonwhites were $5.31(95 \% \mathrm{CI}=1.62,17.4 ; P=0.01)$ and $4.42(95 \% \mathrm{CI}=1.21,16.1 ; P=0.02)$ times as likely to report high chronic pain (Table 3 ). History of inhibitor development was (i) an important confounder and (ii) increased the precision of the model. Thus, it remained in all final parsimonious logistic regression models. 


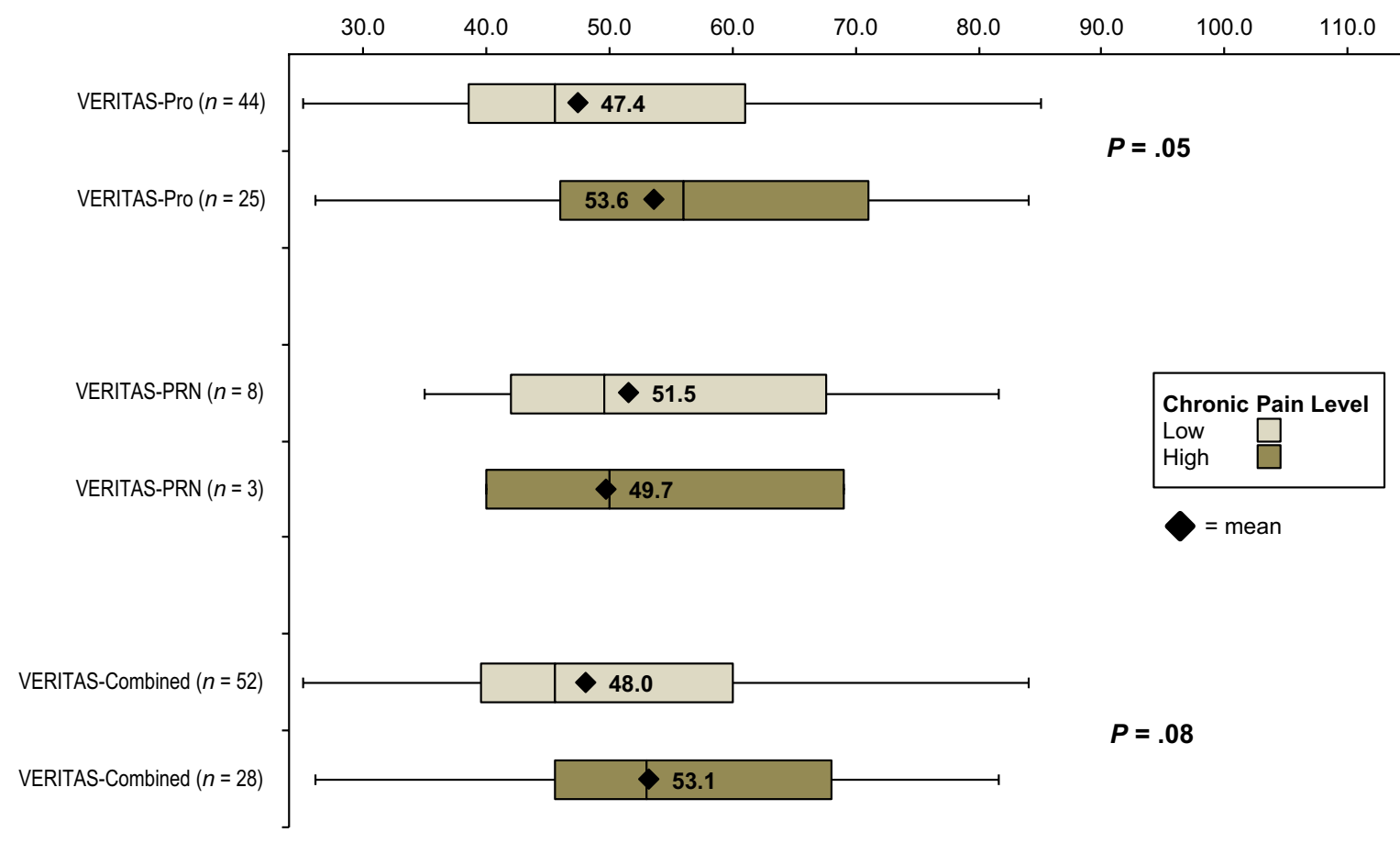

Fig. 1. Box plot of prescribed treatment regimen adherence by chronic pain status $(n=80)$.

Table 2. Logistic regression analyses evaluating the relationship between a 10-point decrease in VERITAS (i.e. increase in adherence to prescribed clottingfactor treatment regimen) and self-reported high (vs. low) levels of chronic pain.

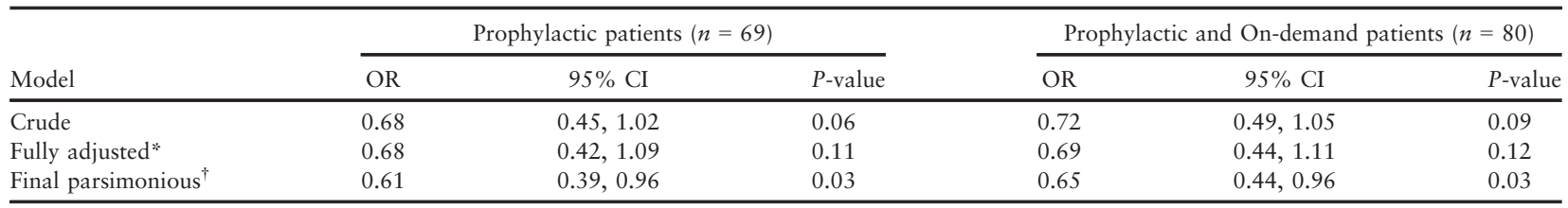

*Fully adjusted model includes VERITAS score, age, race, ethnicity, insurance status, inhibitor development, bleeding disorder type, bleeding disorder severity and history of inhibitor development.

†The final parsimonious model includes VERITAS score, race and history of inhibitor development.

Table 3. Final parsimonious logistic regression models evaluating the relationship between adherence to prescribed clotting-factor treatment regimen and self-reported high (vs. low) levels of chronic pain.

\begin{tabular}{|c|c|c|c|c|c|c|}
\hline \multirow[b]{2}{*}{ Variable } & \multicolumn{3}{|c|}{ Prophylactic patients $(n=69)$} & \multicolumn{3}{|c|}{ Prophylactic and On-demand patients $(n=80)$} \\
\hline & OR & $95 \% \mathrm{CI}$ & $P$-value & OR & $95 \% \mathrm{CI}$ & $P$-value \\
\hline \multicolumn{7}{|l|}{ Increase in adherence } \\
\hline 10-point decrease in VERITAS & 0.61 & $0.39,0.96$ & 0.03 & 0.65 & $0.44,0.96$ & 0.03 \\
\hline \multicolumn{7}{|l|}{ Race } \\
\hline Non-white* & 4.42 & $1.21,16.1$ & 0.02 & 5.31 & $1.62,17.4$ & 0.01 \\
\hline White & 1.00 & & & 1.00 & & \\
\hline \multicolumn{7}{|l|}{ Inhibitor development ${ }^{\dagger}$} \\
\hline Ever & 1.44 & $0.45,4.61$ & 0.54 & 1.31 & $0.44,3.89$ & 0.62 \\
\hline Never & 1.00 & & & 1.00 & & \\
\hline
\end{tabular}

"Most (73\%) of non-white respondents were black or African American, 14\% were mixed race, 9\% were Asian and 5\% were American Indian or Alaskan Native.

'Inhibitor development, though not a statistically significant predictor of having high chronic pain, was included in the final model because of its confounding influence on the primary risk factor of interest (i.e. VERITAS score) and because it increased the precision of the model (i.e. reduced the standard error of VERITAS score parameter).

\section{Discussion}

This study provides evidence that among AYAs with moderate or severe haemophilia, better adherence to prescribed treatment regimens (either prophylactic or on demand) is associated with a significantly lower likelihood of having high levels of chronic pain. Specifically, adjusted logistic regression analysis revealed that for each 10-point reduction (i.e. increase in adherence) in the experimental VERI- 
TAS-combined (Pro and PRN) and the VERITASPro score there was a statistically significant 35\% and $39 \%$ reduction in the odds of having high chronic pain, respectively, among moderate or severe AYA haemophilia patients. Although previous studies have attempted to document the relationship between adherence and health outcomes [8], this is the first study to assess the relationship using a validated scale of adherence. Moreover, this study's primary health outcome of interest (chronic pain) is well-known to be one of the most serious problems afflicting those with haemophilia [14]. Chronic pain has long been known to diminish physical, emotional and social aspects of quality of life and contribute to increased health care utilization and long-term disability [15-19].

Many factors are thought to influence haemophilia treatment adherence, including knowledge about the disease and the importance of treatment, disease severity and the frequency of haemophilia symptoms, treatment satisfaction, frequency of administration, the amount of time spent in an HTC, reminder telephone calls, the age of the PWH and the quality of relationships among patients and their health care professionals $[2,8,20-23]$. Yet, improving adherence still remains a difficult task. Broadly, a previous systematic review revealed that the most successful strategies across several types of chronic disease have included: education with behavioural support, reminder/recall systems and pharmacist-led, multicomponent interventions [24]. On the basis of results from this study that show a relationship between adherence to clotting-factor treatment and level of chronic pain - these strategies, along with other novel approaches, should be continuously evaluated in the haemophilia population, especially in AYAs.

Compared to the general haemophilia population, AYA PWH are unique in that as children transition into young adulthood, their activity level may change, which could affect the risk of bleeding. Likewise, social pressures and/or lifestyle changes may interfere with their adherence to prophylaxis (i.e. prophylaxis may become a lower priority for the patient during adolescence). Moreover, AYAs often begin taking responsibility for self-treatment of haemophilia $[2,25]$. Previous research has suggested that as many as twothirds of young adults will experiment with stopping or reducing prophylactic dosing [26] and that AYA PWH are not only less adherent to prophylaxis but that AYA PWH perceive the need for prophylaxis as unimportant [2]. Although no randomized controlled trials have been done to demonstrate the efficacy of prophylaxis in adolescents, there is evidence that continuing prophylaxis, or starting secondary prophylaxis, reduces the risk of bleeding and helps preserve joint health and quality of life $[27,28]$. This study reinforces the need to provide resource support neces- sary for AYA PWHs to achieve optimal adherence to prescribed clotting-factor treatment regimens - as this may reduce the likelihood of having high levels of chronic pain.

Another interesting (and surprising) finding from this study was that non-white AYA patients with moderate or severe haemophilia were $>4$ times as likely as whites to report high levels of chronic pain. This was true among both prophylactic-only and all patients. This finding is particularly concerning because it remained even after statistical adjustment for demographic (e.g. insurance status and age) and clinical factors (e.g. severity and inhibitor development). Previous research, though not specific to $\mathrm{PWH}$, has shown that African American children have significantly higher postoperative pain scores and require more morphine than white children [29]. A systematic review of racial disparities in pain management supports our findings and suggested that significant racial and ethnic disparities exist in both evaluating pain and providing adequate analgesics. Specifically, racial minorities were more likely to experience greater pain and worse pain management across a variety of health care settings including acute pain managed in the emergency department, postoperative care, childbirth, chronic non-cancer pain, arthritis pain, cancer-related pain and end-of-life care [30]. Moreover, previous research has suggested that compared to whites, blacks with chronic pain experience greater pain severity, more disability and heightened psychosocial problems related to their pain, including depression, post-traumatic stress disorder and anxiety [31,32]. Another report suggested that patients receiving plasma-derived activated prothrombin complex concentrates (pd-aPCC) were more likely to be African American [33], which may suggest that our inhibitor (ever vs. never) designation may not fully control for the impact of inhibitor development. Future research should more thoroughly examine the relationship between race and chronic pain among PWH.

This study has limitations. Primarily, data are crosssectional, thus causal inference cannot be made. Specifically, although study results support that adherence to clotting-factor treatment regimen and level of chronic pain are strongly related among moderate and severe haemophilia AYA patients, the directionality of this relationship cannot be confirmed. That is, though we modelled how self-reported level of adherence impacts the odds of having high chronic pain - it is also possible that level of chronic pain impacts adherence. This cannot be teased out in a cross-sectional study and should be examined in the future with prospective studies. A second limitation is that all data are selfreported. As such, information about blood disorder type and severity, health insurance coverage and other demographic, clinical and behavioural information are not confirmed by medical record review or administra- 
tive claims data. However, by obtaining data through self-report, this study was able to collect important, reliable and valid patient-report outcomes (PRO) data about adherence and chronic pain. Patient-reported data about pain are considered the 'gold standard' and the VERITAS scales are the only validated measures of adherence to clotting-factor treatment developed to date. Another limitation is that the VERITAS-combined score that we reported, though it statistically comprised of two validated instruments, is an experimental, non-validated measure that has not been previously reported. Most participants (86\%), however, treated prophylactically, and VERITAS-Pro scores, which have been previously validated, confirmed the results of the experimental, combined score. Finally, AYA PWH were primarily recruited from large national or regional haemophilia meetings. Thus, our convenience sample of AYA PWH may not adequately represent the broader AYA PWH population who do not typically attend these meetings.

\section{Conclusion}

This study provides evidence that among AYAs with moderate or severe haemophilia, better adherence to prescribed clotting-factor treatment regimens (either prophylactic or on demand) is associated with a significantly lower likelihood of having high levels of chronic pain. As AYA PWH transition into early adulthood and often begin taking responsibility for self-treatment of haemophilia [2] maintaining adherence becomes increasingly important. Future studies should seek to confirm the association between clotting-factor adherence and chronic pain among AYA PWH, evaluate strategies to improve adherence in this group, and to explore why non-whites were more than four times as likely as whites to report high levels of chronic pain.

\section{Acknowledgements}

This study was sponsored and funded by Pfizer Inc.

\section{Disclosures}

Drs. McLaughlin, Anderson, and Tortella are employees and shareholders of Pfizer Inc. Mr. Munn is a member of Nurse Advisory Board for Baxter, Biogen-Idec, CSL Behring, NovoNordisk and Pfizer. He received a consulting fee from Bayer and was a speaker for Novo Nordisk. Dr. Witkop and Mrs. Lambing stated that they had no interests which might be perceived as posing a conflict or bias.

\section{References}

1 Nazzaro AM. Where are we: preliminary results of the national hemophilia foundation's prevention program baseline survey of health knowledge, attitudes, and behaviors in the hemophilia population. HemAware 2000; 20-5: 24-25.

2 Lindvall K, Colstrup L, Wollter IM et al. Compliance with treatment and understanding of own disease in patients with severe and moderate haemophilia. Haemophilia 2006; 12: 47-51.

3 Hacker MR, Geraghty S, Manco-Johnson M. Barriers to compliance with prophylaxis therapy in haemophilia. Haemophilia 2001; 7: 392-6.

4 Blanchette VS, Manco-Johnson M, Santagostino E, Ljung R. Optimizing factor prophylaxis for the haemophilia population: where do we stand? Haemophilia 2004; 10 (Suppl. 4): 97-104.

5 Manco-Johnson MJ. Update on treatment regimens: prophylaxis versus on-demand therapy. Semin Hematol 2003; 40: 3-9.

6 D'Angelo E, Woolf A, Bessette J, Rappaport L, Ciborowski J. Correlates of medical compliance among hemophilic boys. J Clin Psychol 1992; 48: 672-80.

7 Thornburg CD, Pipe SW. Adherence to prophylactic infusions of factor VIII or factor IX for haemophilia. Haemophilia 2006; 12: 198-9.

8 du Treil S, Rice J, Leissinger CA. Quantifying adherence to treatment and its relationship to quality of life in a well-characterized haemophilia population. Haemophilia 2007; 13: 493-501.
9 Thornburg CD. Physicians' perceptions of adherence to prophylactic clotting factor infusions. Haemophilia 2008; 14: 25-9.

10 Duncan N, Kronenberger W, Roberson C, Shapiro A. VERITAS-Pro: a new measure of adherence to prophylactic regimens in haemophilia. Haemophilia 2010; 16: 247-55.

11 Duncan NA, Kronenberger WG, Roberson CP, Shapiro AD. VERITAS-PRN: a new measure of adherence to episodic treatment regimens in haemophilia. Haemophilia 2010; 16: 47-53.

12 Hicks CL, von Baeyer CL, Spafford PA, van Korlaar I, Goodenough B. The Faces Pain Scale-Revised: toward a common metric in pediatric pain measurement. Pain 2001; 93: 173-83.

13 Mickey RM, Greenland S. The impact of confounder selection criteria on effect estimation. Am J Epidemiol 1989; 129: 125 37.

14 Elander J, Robinson G, Mitchell K, Morris $\mathrm{J}$. An assessment of the relative influence of pain coping, negative thoughts about pain, and pain acceptance on health-related quality of life among people with hemophilia. Pain 2009; 145: 169-75.

15 Gibson SJ, Helme RD. Cognitive factors and the experience of pain and suffering in older persons. Pain 2000; 85: 375-83.

16 Gibson SJ, Katz B, Corran TM, Farrell MJ, Helme RD. Pain in older persons. Disabil Rehabil 1994; 16: 127-39.

17 Green CR, Baker TA, Smith EM, Sato Y. The effect of race in older adults presenting for chronic pain management: a comparative study of black and white Americans. $J$ Pain 2003; 4: 82-90.
18 Scudds RJ, McD Robertson J. Empirical evidence of the association between the presence of musculoskeletal pain and physical disability in community-dwelling senior citizens. Pain 1998; 75: 229-35.

19 Weir R, Browne G, Tunks E, Gafni A, Roberts J. Gender differences in psychosocial adjustment to chronic pain and expenditures for health care services used. Clin J Pain 1996; 12: 277-90.

20 Lindvall K, Colstrup L, Loogna K, Wollter I, Gronhaug S. Knowledge of disease and adherence in adult patients with haemophilia. Haemophilia 2010; 16: 592-6.

21 Remor E. Predictors of treatment difficulties and satisfaction with haemophilia therapy in adult patients. Haemophilia 2011; 17: e901-5.

22 De Moerloose P, Urbancik W, Van Den Berg HM, Richards M. A survey of adherence to haemophilia therapy in six European countries: results and recommendations. Haemophilia 2008; 14: 931-8.

23 Gater A, Thomson TA, Strandberg-Larsen M. Haemophilia B: impact on patients and economic burden of disease. Thromb Haemost 2011; 106: 398-404.

24 Viswanathan M, Golin CE, Jones CD et al. Interventions to improve adherence to selfadministered medications for chronic diseases in the United States: a systematic review. Ann Intern Med 2012; 157: 78595.

25 Schrijvers LH, Uitslager N, Schuurmans MJ, Fischer K. Barriers and motivators of adherence to prophylactic treatment in haemophilia: a systematic review. Haemophilia 2013; 19: 355-61. 
512 J. M. MCLAUGHLIN et al.

26 Fischer K, Valentino L, Ljung R, Blanchette V. Prophylaxis for severe haemophilia: clinical challenges in the absence as well as in the presence of inhibitors. Haemophilia 2008; 14(Suppl. 3): 196-201.

27 Collins P, Faradji A, Morfini M, Enriquez MM, Schwartz L. Efficacy and safety of secondary prophylactic vs. on-demand sucrose-formulated recombinant factor VII treatment in adults with severe hemophilia A: results from a 13-month crossover study. I Thromb Haemost 2010; 8: 83-9.

28 Valentino LA. Secondary prophylaxis therapy: what are the benefits, limitations and unknowns? Haemophilia 2004; 10: 14757.

29 Sadhasivam S, Chidambaran V, Ngamprasertwong $\mathrm{P}$ et al. Race and unequal burden of perioperative pain and opioid related adverse effects in children. Pediatrics 2012, 129: 832-8.

30 Anderson KO, Green CR, Payne R. Racial and ethnic disparities in pain: causes and consequences of unequal care. The journal of pain: official journal of the American Pain Society 2009; 10: 1187-204.

31 Green CR, Ndao-Brumblay SK, Nagrant AM, Baker TA, Rothman E. Race, age, and gender influences among clusters of African American and white patients with chronic pain. J Pain 2004; 5: 171-82.

32 Green CR, Hart-Johnson T. The adequacy of chronic pain management prior to presenting at a tertiary care pain center: the role of patient socio-demographic characteristics. I Pain 2010; 11: 746-54.

33 Pokras SM, Petrilla AA, Weatherall J, Lee WC. The economics of inpatient ondemand treatment for haemophilia with high-responding inhibitors: a US retrospective data analysis. Haemophilia 2012; 18: 284-90. 